\title{
Pseudogap and Fermi-arc evolution in the phase-fluctuation scenario
}

\author{
Qiang Han, ${ }^{1,2}$ Tao Li, ${ }^{1}$ and Z. D. Wang ${ }^{2}$ \\ ${ }^{1}$ Department of Physics, Renmin University of China, Beijing, China \\ ${ }^{2}$ Department of Physics and Center of Theoretical and Computational Physics, The University of Hong Kong, Pokfulam Road, \\ Hong Kong, China
}

(Received 6 July 2010; published 27 August 2010)

\begin{abstract}
Pseudogap phenomena and the formation of Fermi arcs in underdoped cuprates are numerically studied in the presence of phase fluctuations that are simulated by an $X Y$ model. Most importantly the spectral function for each Monte Carlo sample is calculated directly and efficiently by the Chebyshev polynomials without having to diagonalize the fermion Hamiltonian, which enables us to handle a system large enough to achieve sufficient momentum/energy resolution. We find that the momentum dependence of the energy gap is identical to that of a pure $d$-wave superconductor well below the Kosterlitz-Thouless $(\mathrm{KT})$ transition temperature $\left(T_{\mathrm{KT}}\right)$ while displays an upturn deviation from $\cos k_{x}-\cos k_{y}$ with increasing temperature. An abrupt onset of the Fermi arcs is observed above $T_{\mathrm{KT}}$ and the arc length exhibits a similar temperature dependence to the thermally activated vortex excitations.
\end{abstract}

DOI: 10.1103/PhysRevB.82.052503

PACS number(s): 74.72.-h, 74.20.Rp, 74.25.Jb

One of the most important open issues in the research of high-temperature superconductivity is the origin of the socalled pseudogap and its relation to the superconducting gap. One viewpoint regards the pseudogap as a preformed Cooper-pair state without long-range phase coherence, yet another attributes its origin to a new phase of matter. The most direct evidence of the pseudogap in hole-doped cuprates comes from experimental techniques such as angle resolved photoemission spectroscopy (ARPES) and scanning tunneling spectroscopy (STS), which measure the lowenergy single-particle spectrum and relate the loss of spectral weight to the pseudogap opening. ${ }^{1-3}$ One peculiar property of the pseudogap phase revealed by ARPES is the truncated Fermi surfaces termed as "Fermi arcs." 4 The Fermi arcs emerge abruptly around the nodal region above $T_{c}$, and extend out to the Brillouin-zone edge with increasing temperature and form a complete Fermi surface for temperature above $T^{*}$. $4-6$

The evolution of the Fermi surface in the pseudogap phase has been addressed ${ }^{7-9}$ within the phase fluctuation context. The formation of Fermi arcs was attributed to the pileup of low-energy spectral weight due to the Doppler effect of the fluctuating superjacent. ${ }^{7}$ However such analytic results were merely based on a semiclassical approximation ${ }^{10}$ which was argued to be uncontrolled ${ }^{11}$ and the momentum and temperature dependence of the energy gap was not addressed. Numerical treatment of the effect of phase fluctuations involved a combination of the Monte Carlo (MC) simulation of the Landau-Ginzburg free energy and the exact diagonalization of the fermion Hamiltonian. ${ }^{8}$ However, the competition between the superconducting and antiferromagnetic order parameters in the presence of quenched disorder was complicated and the momentum resolution was relative low due to the time-consuming numerical diagonalization procedure.

In this work, we (1) examine the momentum and temperature dependence of the spectral gap and (2) temperature dependence of the Fermi-arc length within the phasefluctuating scenario. The single-electron spectral function is numerically calculated by the Chebyshev polynomial approach to achieve high momentum and energy resolutions.
The fluctuating phases are simulated phenomenologically by a classical $X Y$ model. ${ }^{12,13}$ The two-dimensional $X Y$ free energy is

$$
F\left(\left\{\varphi_{\mathbf{i}}\right\}\right)=-\frac{J}{2} \sum_{\mathbf{i}, \delta} \cos \left(\varphi_{\mathbf{i}}-\varphi_{\mathbf{i}+\delta}\right),
$$

where the phase-stiffness constant $J$ is the energy scale of the Kosterlitz-Thouless transition. $\varphi_{\mathbf{i}}$ denotes the phase of the superconducting order parameter defined on the lattice site $\mathbf{i}$. $\boldsymbol{\delta}$ is the unit vector along $x$ or $y$ direction connecting $\mathbf{i}$ to its nearest neighbors $(\mathrm{NN})$. The Hamiltonian of noninteracting electrons moving in a spatially fluctuating pairing field given by Eq. (1) reads

$$
\begin{aligned}
\hat{H} & =\sum_{\mathbf{i}, \mathbf{j}, \sigma}\left(-t_{\mathbf{i} \mathbf{j}}-\mu \delta_{\mathbf{i j}}\right) c_{\mathbf{i} \sigma}^{\dagger} c_{\mathbf{j} \sigma}+\sum_{\mathbf{i}, \mathbf{j}}\left(\Delta_{\mathbf{i j}} c_{\mathbf{i} \uparrow}^{\dagger} c_{\mathbf{j} \downarrow}^{\dagger}+\text { H.c. }\right), \\
& =\sum_{\mathbf{i}, \mathbf{j}}\left(c_{\mathbf{i} \uparrow}^{\dagger}, c_{\mathbf{i} \downarrow}\right)\left(\begin{array}{cc}
-t_{\mathbf{i} \mathbf{j}}-\mu \delta_{\mathbf{i j}} & \Delta_{\mathbf{i j}} \\
\Delta_{\mathbf{i} \mathbf{j}}^{*} & t_{\mathbf{i j}}+\mu \delta_{\mathbf{i} \mathbf{j}}
\end{array}\right)\left(\begin{array}{c}
c_{\mathbf{j} \uparrow} \\
c_{\mathbf{j} \downarrow}^{\dagger}
\end{array}\right),
\end{aligned}
$$

where $t_{\mathrm{ij}}$ denotes hopping integral and $\mu$ represents the chemical potential. The pairing potential $\Delta_{\mathrm{ij}}$ is defined on the $\mathrm{NN}$ bond $(\mathbf{i}, \mathbf{j}=\mathbf{i}+\boldsymbol{\delta})$. Here we fix the amplitude of the pairing potential while concentrate on the role played by the phase fluctuation, i.e., $\Delta_{\mathbf{i}, \mathbf{i}+\delta}=\Delta e^{i \theta_{\delta}^{(\mathbf{i})}}$ with $\Delta$ kept constant. We use the expression $\theta_{\hat{x}, \hat{y}}(\mathbf{i})= \pm \operatorname{Im} \ln \left(e^{i \varphi_{\mathbf{i}}}+e^{i \varphi_{\mathbf{i}+\delta}}\right)$ to relate the phase of the pairing potential with $\varphi_{\mathbf{i}}$, which gives rise to a pure $d$-wave pairing state in the low-temperature limit when the thermal fluctuation is negligible small.

In our numerical study, a set of phase configurations $\left\{\varphi_{\mathbf{i}}\right\}$ is first generated by the classical Monte Carlo simulation according to the probability distribution $\exp \left\{-F\left[\left(\varphi_{\mathbf{i}}\right)\right] / T\right\}$. Then the single-electron spectral function of the Bogoliubov-de Gennes (BdG) Hamiltonian (2) for each individual phase configuration, which is denoted by $A_{\varphi}(\mathbf{k}, \omega)$ in short, is calculated and at last averaged over the sampled phase space to obtain the expectation value of the spectral function $A(\mathbf{k}, \omega)=\left\langle A_{\varphi}(\mathbf{k}, \omega)\right\rangle$. The bottleneck in this algo- 
rithm mainly lies in the numerical calculation of the spectrum of the BdG Hamiltonian (2). Previous studies ${ }^{8,12}$ attempted to solve the problem by direct diagonalization of the BdG Hamiltonian, whose workload is $O\left(N^{3}\right)$. In this paper, we deploy the Chebyshev expansion approach ${ }^{14}$ to directly calculate the spectral function, which circumvents the difficulty in fully diagonalizing a large Hermitian matrix. As illustrated in the following, the computational load for each $A_{\varphi}(\mathbf{k}, \omega)$ is approximately $O(M N)$ with $M \ll N$, which is a significant advance regarding to numerical studies of the above problem.

The single-particle spectral function corresponding to the quadratic Hamiltonian of Eq. (2) can be calculated from the imaginary part of the Green's function

$$
A_{\varphi}(\nu, \omega)=-\operatorname{Im}\left\langle\nu\left|\left(\omega+i \eta-\hat{H}_{\varphi}\right)^{-1}\right| \nu\right\rangle / \pi .
$$

Here more generally, $|\nu\rangle$ denotes any single-particle wave vector concerned with. $|\nu\rangle$ can be chosen as $|\mathbf{k}\rangle=\frac{1}{\sqrt{N}} \sum_{\mathbf{i}} e^{i \mathbf{k} \cdot \mathbf{i}}|\mathbf{i}\rangle$ with $\mathbf{k}$ the momentum in the first Brillouin zone, and accordingly the obtained $A(\mathbf{k}, \omega)$ are related to the evolution of the Fermi surface as observed by ARPES. If $|\nu\rangle=|\mathbf{i}\rangle, A(\mathbf{i}, \omega)$ is actually the local density of state related to the STS measurements. According to the kernel polynomial method, ${ }^{14}$ $A_{\varphi}(\nu, \omega)$ can be expanded in truncated series of the Chebyshev polynomials of the first kind $T_{m}$

$$
A_{\varphi}(\nu, \omega)=-\frac{\mu_{0}+2 \sum_{m=1}^{M-1} \mu_{m} T_{m}(\omega / s)}{\pi \sqrt{1-(\omega / s)^{2}}},
$$

where the expansion coefficients $\mu_{m}$ $=\left\langle\nu\left|T_{m}(\hat{H} / s)\right| \nu\right\rangle \sinh [\lambda(1-m / M)] / \sinh (\lambda)$ are Chebyshev moments. The Lorentz kernel factor is used to improve the convergence of the truncated series and damps Gibbs oscillations. A good value of the kernel parameter is $\lambda=4$. The scaling factor $s$ fits the spectrum of the Hamiltonian $\hat{H} / s$ into the interval $[-1,1]$. We choose $s$ to be slightly larger than the bandwidth of the BdG Hamiltonian (2). The energy resolution of the spectral function is estimated by $\varepsilon=\lambda s / M$. Most computational effort is spent in the calculation of the moments $\mu_{m}$, which reduces to matrix-vector multiplications after taking advantage of the recursion relation $T_{m}(x)$ $=2 x T_{m-1}(x)-T_{m-2}(x)$. Considering that the BdG Hamiltonian (2) is sparse, the cost of matrix-vector multiplication is an order $O(N)$ process and the calculation of $M$ moments requires only $O(M N)$ computational operations, which even enables us to handle larger lattices using a desktop computer. Furthermore, recursive relations of Chebyshev polynomials $T_{2 m}=2 T_{m}^{2}-1$ and $T_{2 m+1}=2 T_{m} T_{m+1}-T_{1}$ enable us to obtain two moments per matrix-vector multiplication.

We now present the results of our simulations. Typical calculation is done on a $96 \times 96$ lattice. Combined Monte Carlo method is used to generate equilibrium configurations of phase angles $\left\{\varphi_{i}\right\}$. The KT-phase transition is observed with $T_{\mathrm{KT}} \approx J$ by studying the phase correlation and the vortex density as a function of temperature, which is consistent with our previous work. ${ }^{9}$ For each temperature, the first $10^{5} \mathrm{MC}$ sweeps are dropped to equilibrate the system. $10^{3}$ phase con-

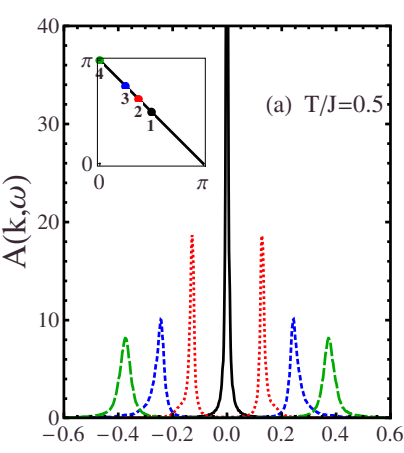

$\omega / \mathrm{t}$

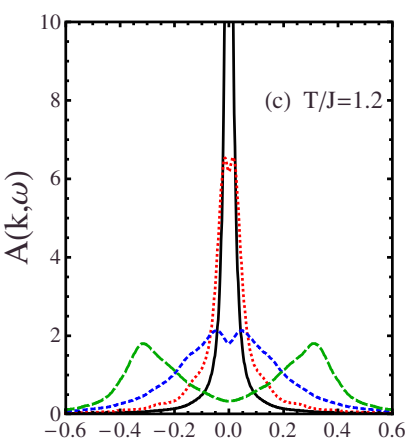

$\omega / \mathrm{t}$

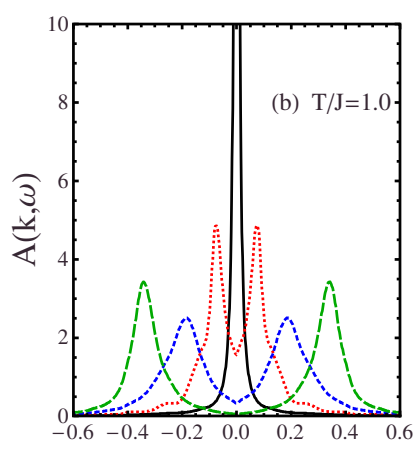

$\omega / \mathrm{t}$

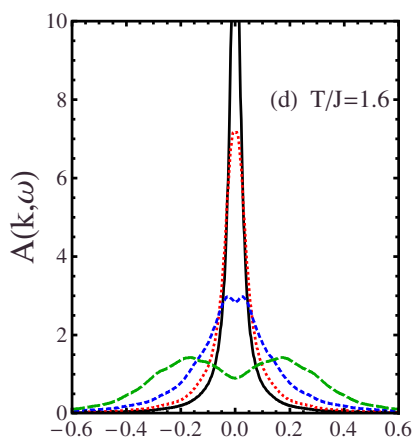

$\omega / \mathrm{t}$
FIG. 1. (Color online) Energy and temperature dependence of the spectral functions $A(\mathbf{k}, \omega)$ at four Fermi momenta on the Fermi surface in first quarter of the first Brillouin zone for illustration. Black solid line (first k), red dotted line (second $\mathbf{k}$ ), blue shortdashed line (third $\mathbf{k}$ ), and green long-dashed line (fourth $\mathbf{k}$ ). (a) at $T / J=0.5$ in the superconducting state; (b) at $T / J=1.0$ around the KT phase transition; and $[(\mathrm{c})$ and (d)] at $T / J=1.2,1.6$ in the pseudogap state.

figurations every $10^{3} \mathrm{MC}$ sweeps are used to get thermal averages, which are sufficient to reduce the statistical errors. For each phase configuration, the corresponding spectral function is calculated by Chebyshev expansions with the truncation $M=4096$, which results in an approximate energy resolution $\varepsilon / t \simeq 0.004$. Here $t$ denotes the NN hopping integral. Most calculations are performed for the particle-hole symmetric case $\left(t^{\prime}=\mu=0\right)$ for simplicity. $\Delta=0.1 t$ which results in the superconducting gap $0.4 t$ for the particle-hole symmetric case in the zero temperature limit. The resulting Fermi surface has a diamond shape, part of which is shown in the inset of Fig. 1(a). On the node-to-antinode segment of the Fermi surface, 49 equally spaced Fermi momenta are chosen to achieve sufficient momentum resolution.

Figure 1 shows the energy distribution of the spectral function $A(\mathbf{k}, \omega)$ for several Fermi momenta for illustration. At $T / J=0.5$ we find sharp spectral peaks, indicating welldefined Bogoliubov quasiparticle excitations. Furthermore the superconducting gap vanishes only at the node, away from which the gap gradually opens and increases to its full size at the antinodal point. With increasing temperature from Figs. 1(a)-1(d), the spectral lines are broadened due to the strengthened scattering effect from the pair fluctuations. The energy gap for $\mathbf{k}$ at the antinode (see the evolution of the green long-dashed curves) keeps opening while that near the node [see red dotted curves of Figs. 1(a)-1(d) and 2(d)] 

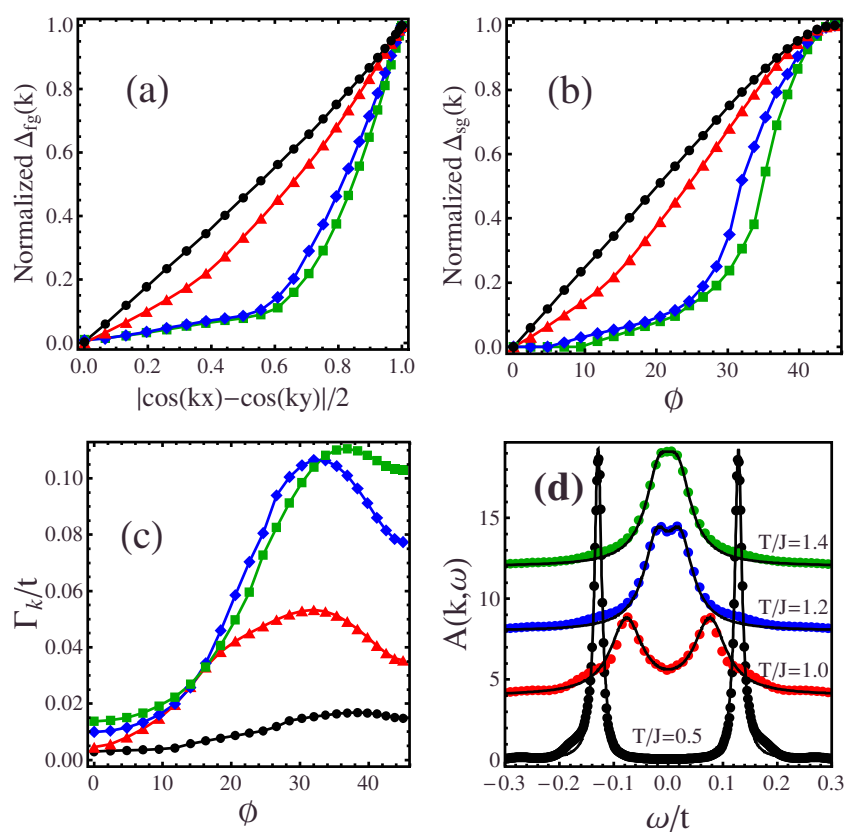

FIG. 2. (Color online) [(a) and (b)] momentum dependence of energy gap at $T / J=0.5,1.0,1.2,1.4$ (black dot, red triangle, blue diamond, and green square). (a) The fitted gap [see Eq. (5)] plotted as a function of $\left|\cos \left(k_{x}\right)-\cos \left(k_{y}\right)\right| / 2$. (b) The spectral gap (half the peak-to-peak distance) plotted as a function of $\phi$, i.e., the angle of $\mathbf{k}$ relative to the nodal point. Both gaps are normalized to their values at the antinode. (c) Momentum dependence of $\Gamma_{\mathbf{k}}$ [see Eq. (5)] and (d) $A(\mathbf{k}, \omega)$ with $\mathbf{k}$ corresponding to the red dot in the inset of Fig. 1(a), superimposed by their fits (black lines) according to Eq. (5).

closes as temperature rises across $T_{\mathrm{KT}} \approx J$, indicating the formation of pseudogap at the antinodal region and the growth of Fermi arc near the nodal region above $T_{\mathrm{KT}}$. From the calculated spectral function in momentum-energy space, one can suggest two types of gap: the spectral gap $\Delta_{s g}(\mathbf{k})$ which is half of the peak-peak separation ${ }^{15}$ and the fitted gap $\Delta_{f g}(\mathbf{k})$ by numerical fitting with a simple phenomenological model $A(\mathbf{k}, \omega)=-\operatorname{Im}\left[\omega-\xi_{\mathbf{k}}-\Sigma(\mathbf{k}, \omega)\right]^{-1} / \pi$ with the modified BCS self-energy ${ }^{16-18}$

$$
\Sigma(\mathbf{k}, \omega)=-i \Gamma_{\mathbf{k}}+\frac{\Delta_{f g}^{2}(\mathbf{k})}{\omega+\xi_{\mathbf{k}}+i \Gamma_{\mathbf{k}}},
$$

where $\quad \xi_{\mathbf{k}}=-2 t\left(\cos k_{x}+\cos k_{y}\right)-4 t^{\prime} \cos k_{x} \cos k_{y}-\mu \quad$ the normal-state electron dispersion relation. $\Delta_{f g}(\mathbf{k})$ and $\Gamma_{\mathbf{k}}$ are fitting parameters. $\Gamma_{\mathbf{k}}$ takes into account of the lifetime broadening due to the scattering effect from the pair fluctuations. Our model of a momentum-dependent scattering rate $\Gamma_{\mathbf{k}}$ is more general than that considered in Refs. 17 and 18. Figure 2(d) shows the typical fitting results together with the raw data calculated from Monte Carlo simulations.

Figures 2(a) and 2(b) show the dispersion of the fitted gap $\Delta_{f g}(\mathbf{k})$ and spectral gap $\Delta_{s g}(\mathbf{k})$. At $T / J=0.5$ (and below) both gap functions show simple $d_{x^{2}-y^{2}}$ form, $\left|\cos k_{x}-\cos k_{y}\right| / 2$, indicating that the effect of the pair fluctuation is rather weak well below $T_{\mathrm{KT}}$. With increasing temperature until $T / J=1.0$ which is approximately equal to $T_{\mathrm{KT}}$, both gap functions de-
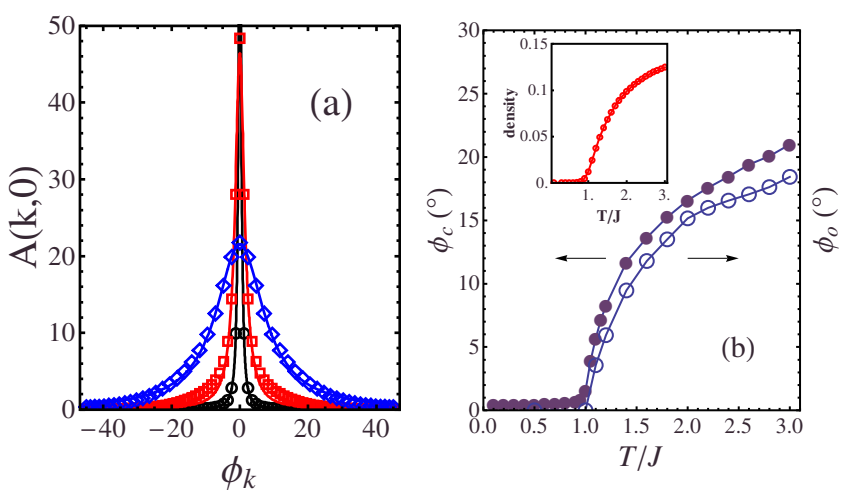

FIG. 3. (Color online) (a) Momentum dependence of $A(\mathbf{k}, \omega$ $=0)$ with $\mathbf{k}$ along the underlying Fermi surface at $T / J$ $=0.5,1.0,1.2$ (black circle, red square, and blue diamond). $\phi_{\mathbf{k}}$ denotes the angle of $\mathbf{k}$ relative to the node point. Solid colored lines are fits to a Lorentzian-type function $\frac{a}{\phi_{\mathbf{k}}^{2}+\phi_{c}^{2}}$. (b) $\phi_{c}$ and $\phi_{o}$ as a function of temperature. The inset shows the temperature dependence of vortex density.

viate from the pure $d$-wave form and exhibit an upturn behavior due to increased spin-wave-type superconducting fluctuations. This deviation increases more rapidly with temperature from $T / J=1.0$ to 1.2 , which indicates that the vortexlike excitations are more efficient in Doppler shifting the single-electron spectral functions. From Fig. 2(a), the behavior of $\Delta_{f g}$ changes gradually with temperature. However by examining $\Delta_{s g}$ as in shown in Fig. 2(b), we find that an emergent "arc," i.e., a Fermi segment with zero spectral gap, arises abruptly around $T_{\mathrm{KT}}$ and grows rapidly with temperature from $T / J=1.0$ to 1.2 . This expansion of a Fermi node (below $T_{\mathrm{KT}}$ ) to an Fermi arc (above $T_{\mathrm{KT}}$ ) can be attributed to thermal proliferation of vortices. Figure 2(c) shows the scattering rate as functions of $\mathbf{k}$ and temperature. $\Gamma_{\mathbf{k}}$ increases with temperature due to enhanced pair fluctuations especially near the nodal and antinodal region. Furthermore, above $T_{\mathrm{KT}}$ in the pseudogap state, the scattering rate shows strong momentum anisotropy with minimum at the node. These results are qualitatively consistent with experimental observations. ${ }^{19}$

The length of Fermi arc can be quantified by different ways. One can locate the specific angle $\phi_{o}$ at which the spectral gap begins to open by examining the $\mathbf{k}$ dependence of $\Delta_{s g}(\mathbf{k})$ as indicated in Fig. 2(b). The fact that Fermi arcs are segments of Fermi surface where the spectral function at the Fermi energy has nonvanishing value can also be utilized to trace the evolution. Therefore $A(\mathbf{k}, \omega=0)$ is examined with $\mathbf{k}$ along the underlying Fermi surface as shown in Fig. 3(a). At $T / J=0.5$ we find a sharp peak around the nodal point, which decays rapidly along the node-to-antinode direction indicating pointlike Fermi surface in the superconducting state. This peak is broadened with increasing temperature and accordingly phase fluctuations. We find that a characteristic length $\phi_{c}$ can be extracted by fitting $A(\mathbf{k}, 0)$ with a Lorentzian-type function $\frac{a}{\phi_{\mathbf{k}}^{2}+\phi_{c}^{2}}$, where $\phi_{\mathbf{k}}$ is the angle of $\mathbf{k}$ relative to the node. This is not accidental but compatible with Eq. (5). For $\mathbf{k}$ along the Fermi surface and near the gap node, $\Delta_{f g}(\mathbf{k}) \approx\left(\sqrt{2} \pi-k_{F}\right) v_{\Delta} \phi_{\mathbf{k}}$ and after considering that $\xi_{\mathbf{k}}$ $=0$ we have according to Eq. (5) 


$$
A(\mathbf{k}, 0)=\frac{\Gamma_{\mathbf{k}} / \pi}{\Delta_{f g}^{2}(\mathbf{k})+\Gamma_{\mathbf{k}}^{2}} \approx \frac{a}{\phi_{\mathbf{k}}^{2}+\phi_{c}^{2}} .
$$

Figure 3(b) shows the temperature dependence of $\phi_{c}$ and $\phi_{o}$ obtained by examining $A(\mathbf{k}, 0)$ and $\Delta_{s g}(\mathbf{k})$, respectively. Below $T_{\mathrm{KT}}$ the arc length is negligible small, showing that the Fermi node is protected against weak phase fluctuations. Near $T / J=1.0$ there is an apparent jump of $\phi_{o}$ and $\phi_{c}$, indicating that the Fermi arc emerges immediately above $T_{\mathrm{KT}}$ together with the loss of phase coherence, which is consistent with experimental observation. ${ }^{5}$ The relation of Fermi arc formation to the vortex proliferation is illustrated by the inset of Fig. 3(b), where the temperature dependence of the vortex density is shown. The formation of vortices increases rapidly for temperatures near and above $T_{\mathrm{KT}}$ and exhibits a similar temperature behavior to the arc length indicating that vortex-type phase fluctuations are responsible for the formation and evolution of the Fermi surface. This result is qualitatively consistent with an earlier theoretical study ${ }^{7}$ in which the arc length is related to the characteristic width of the supercurrent distribution. However, our work has taken both the Doppler effect of the whirling supercurrent and the scattering effect of vortex centers into full consideration.

In Fig. 4 we plot the intensity of the spectral function at zero energy as a function of $k_{x}$ and $k_{y}$ in the first quarter of the first Brillouin zone for a realistic hopping parameter with $t^{\prime} / t=-0.3$. Well below the KT-transition temperature with ignorable phase fluctuations, the Fermi surface is pointlike as in ideal $d$-wave superconductors. With increasing temperature we find arclike Fermi surface with monotonically increasing arc length, which is qualitatively consistent with ARPES observation. 5,6

In summary, the influence of thermal phase fluctuation on the single-particle spectral function has been studied via numerical techniques which combine the Monte Carlo method with the Chebyshev expansion approach. The dispersion of the spectral gap deviates from the simple $d$-wave form especially for $T>T_{\mathrm{KT}}$ when vortexlike fluctuations are dominant.
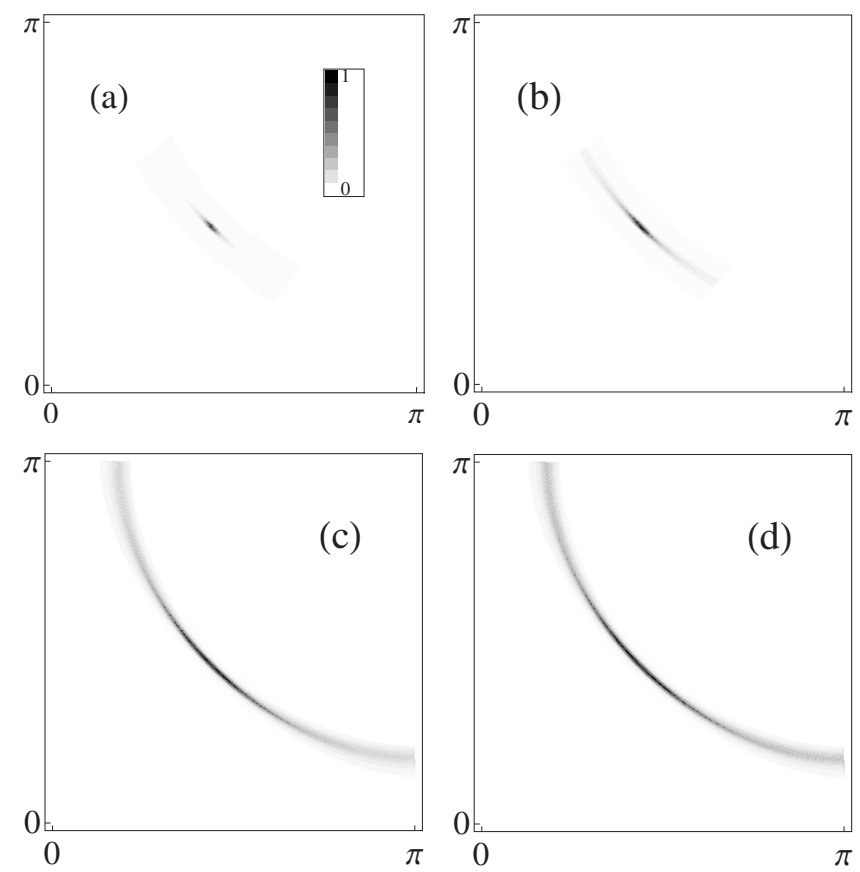

FIG. 4. The temperature dependence of $A(\mathbf{k}, \omega=0)$ in the first quarter of the first Brillouin zone, normalized to its value at the node point for realistic band parameters $t^{\prime} / t=-0.3$. From (a)-(d), $T / J=0.5,1.0,1.6,2.0$.

Apparent onsets of Fermi arcs are found near and above $T_{\mathrm{KT}}$ with the arc length rises monotonically with temperature and displays close relevance to the vortex density. Our results give qualitative understanding of the ARPES observations of the pseudogap phase and Fermi arcs in cuprates.

We thank T. K. Lee and Y. Chen for helpful discussions, and A. Tsvelik for communication. The work was supported by the RGC of Hong Kong under Grants No. HKU7055/09P and No. HKUST3/CRF/09, the URC fund of HKU, the Natural Science Foundation of China under Grant No. 10674179.
${ }^{1}$ D. S. Marshall et al., Phys. Rev. Lett. 76, 4841 (1996).

${ }^{2}$ A. G. Loeser, Z.-X. Shen, D. S. Dessau, D. S. Marshall, C. H. Park, P. Fournier, and A. Kapitulnik, Science 273, 325 (1996).

${ }^{3}$ H. Ding, T. Yokoya, J. C. Campuzano, T. Takahashi, M. Randeria, M. R. Norman, T. Mochiku, K. Kadowaki, and J. Giapintzakis, Nature (London) 382, 51 (1996).

${ }^{4}$ M. R. Norman et al., Nature (London) 392, 157 (1998).

${ }^{5}$ A. Kanigel, U. Chatterjee, M. Randeria, M. R. Norman, S. Souma, M. Shi, Z. Z. Li, H. Raffy, and J. C. Campuzano, Phys. Rev. Lett. 99, 157001 (2007).

${ }^{6}$ A. Kanigel et al., Nat. Phys. 2, 447 (2006).

${ }^{7}$ E. Berg and E. Altman, Phys. Rev. Lett. 99, 247001 (2007).

${ }^{8}$ G. Alvarez and E. Dagotto, Phys. Rev. Lett. 101, 177001 (2008).

${ }^{9} \mathrm{~T}$. Li and Q. Han, arXiv:1003.1783 (unpublished).

${ }^{10}$ M. Franz and A. J. Millis, Phys. Rev. B 58, 14572 (1998).

${ }^{11}$ M. Khodas and A. M. Tsvelik, Phys. Rev. B 81, 094514 (2010).
${ }^{12}$ T. Eckl, D. J. Scalapino, E. Arrigoni, and W. Hanke, Phys. Rev. B 66, 140510(R) (2002).

${ }^{13}$ W. Kim, Y. Chen, and C. S. Ting, arXiv:0809.4273 (unpublished).

${ }^{14}$ A. Weiße, G. Wellein, A. Alvermann, and H. Fehske, Rev. Mod. Phys. 78, 275 (2006).

${ }^{15}$ R. Damascelli, Z. Hussain, and Z.-X. Shen, Rev. Mod. Phys. 75, 473 (2003).

${ }^{16}$ J. R. Schrieffer, Theory of Superconductivity (W. A. Benjamin, New York, 1964).

${ }^{17}$ M. R. Norman, M. Randeria, H. Ding, and J. C. Campuzano, Phys. Rev. B 57, R11093 (1998).

${ }^{18}$ M. R. Norman, A. Kanigel, M. Randeria, U. Chatterjee, and J. C. Campuzano, Phys. Rev. B 76, 174501 (2007).

${ }^{19}$ T. Valla, A. V. Fedorov, P. D. Johnson, Q. Li, G. D. Gu, and N. Koshizuka, Phys. Rev. Lett. 85, 828 (2000). 